\title{
First Measurement of the Production of $\mathbf{K}^{* \pm}$ in Deep Inelastic ep Scattering at HERA
}

\author{
Deniz SUNAR \\ on behalf of the H1 Collaboration \\ University of Antwerpen - Department of Physics \\ Groenenborgerlaan 171-U B-2020 Antwerpen - Belgium
}

\begin{abstract}
A first measurement of the production of $K^{*}(892)^{ \pm}$vector mesons, observed through the decay chain $K^{*}(892)^{ \pm} \rightarrow K_{S}^{0} \pi^{ \pm}$, in neutral current deep-inelastic ep scattering with the $\mathrm{H} 1$ detector is presented [1]. The analysis is based on data taken on the 2005-2007 data taken with the total integrated luminosity of $302 \mathrm{pb}^{-} 1$. Inclusive cross sections are presented as a function of the various kinematic variables. The phase space of the measurement is restricted to $5<Q^{2}<100 \mathrm{GeV}^{2}, 0.1<y<0.6,-1.5<\eta\left(K^{* \pm}\right)<1.5$ and $p_{T}\left(K^{* \pm}\right)>1 \mathrm{GeV}$. The results are compared to predictions of leading order Monte Carlo models with parton showers.
\end{abstract}

\section{Introduction}

The production of strange hadrons in high energy particle collisions provides an opportunity to investigate the strong interactions both in the perturbative and non-perturbative regimes. The cross sections of this analysis represent the first measurement of strange vector meson $K^{* \pm}(892)$ production in deep inelastic scattering (DIS) at HERA. The measurement allows to study the strange content of the proton as well as the processes involved in the production of strange hadrons. Strange quarks can be produced via various subprocesses such as directly in the hard scattering off a strange sea quark inside the proton, via boson gluon fusion and via decays of heavier quarks. The dominant production mechanism of strange quarks is expected to be the string fragmentation. Furthermore, a difference in the $K^{*+}$ and $K^{*-}$ cross sections would indicate an asymmetric distribution of the sea quarks in the proton. The measurement gives direct information about the strangeness suppression factor $\left(\lambda_{s}=P(s) / P(u)\right)$ which describes the probability of strange $(s)$ quark production $P(s)$ relative to the probability of up $(u)$ and down $(d)$ quark production $P(u)=P(d)$.

\section{Selection of Events}

The data presented were collected with the H1 detector at HERA in the years 2005 to 2007, when HERA was colliding 27.5 GeV electrons (or positrons) and $920 \mathrm{GeV}$ protons, yielding a centre-of-mass energy of $\sqrt{s}=318 \mathrm{GeV}$. The results are based on the sum of the $e^{-} p$ and $e^{+} p$ samples, corresponding to an integrated luminosities of $167 \mathrm{pb}^{-1}$ and $135 \mathrm{pb}^{-1}$, respectively.

The kinematic variables $Q^{2}$ and the inelasticity $y$ are determined using the electronsigma method [2]. DIS offline event selection is based on the following requirements. The scattered electron energy $E_{e}$ is required to be $E_{e}^{\prime}>11 \mathrm{GeV}$. The four momentum transfer squared $Q^{2}$ and the in-elasticity $y$ are required to lie in the ranges $5<Q^{2}<100 \mathrm{GeV}^{2}$ and $0.1<y<0.6$.

DIS 2009 


\section{Reconstruction of $\mathrm{K}^{* \pm}$ Mesons}

The first stage in the $K^{* \pm} \rightarrow K_{S}^{0} \pi^{ \pm}$selection process is the identification of neutral kaons. The search for the $K_{S}^{0}$ is performed via the decay $K_{S}^{0} \rightarrow \pi^{+} \pi^{-}$. Two oppositely charged central tracks which originate from a common secondary vertex are considered as $K_{S}^{0}$ decay products. Each track is required to have a minimum transverse momentum $p_{T}$ with respect to the beam direction $p_{T}>120 \mathrm{MeV}$ and pseudo-rapidity $|\eta|<1.5$ to ensure that the track is well contained within the acceptance of the central tracking detector. For further analysis, only $K_{S}^{0}$ candidates with a reconstructed mass window of $470<M\left(\pi^{+} \pi^{-}\right)<520 \mathrm{MeV}$ around the nominal mass of $497.6 \mathrm{MeV}$ are retained. The invariant mass spectra of $K_{S}^{0}$ candidates is shown in Fig. 1 . The signal distribution is fitted with a student's t function while a polynomial function is used for fitting the background distribution. The number of $K_{S}^{0}$ mesons from the fit is $1.32 \times 10^{6}$.

A third charged track which is assumed to be a pion is combined with the $K_{S}^{0}$ candidate in order to construct a $K^{* \pm}$ candidate. The third charged track is required to originate from the primary vertex and to fulfil $p_{T}>120 \mathrm{MeV}$ and $|\eta|<1.5$. The PDG value of the $K_{S}^{0}$ mass is assigned to the $K_{S}^{0}$ candidate and additional cuts are applied on the combination of $K_{S}^{0}$ and $\pi^{ \pm}$, $p_{T}\left(K_{S}^{0} \pi^{ \pm}\right)>1 \mathrm{GeV}$ and $\left|\eta\left(K_{S}^{0} \pi^{ \pm}\right)\right|<1.5$. Finally, the invariant mass of the $K^{* \pm}$ candidates is obtained. After applying the selection cuts described above, the distribution of the invariant mass of the $K^{* \pm}$ candidates is still governed by the large combinatorial background.

The mass distribution of the signal is described with the relativistic Breit-Wigner function. The invariant mass spectra of $K_{S}^{0} \pi^{ \pm}$combinations is shown in Fig. 2. The number of $K^{* \pm}$ mesons from the fit is about 79000 .

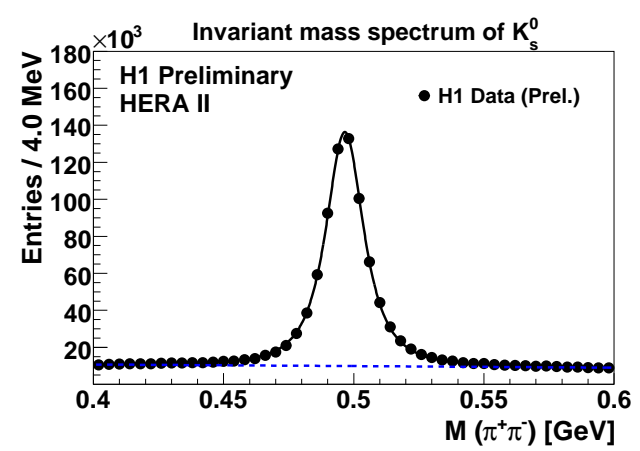

Figure 1: Invariant mass spectrum of the $K_{S}^{0} \rightarrow \pi^{+} \pi^{-}$candidates. The points correspond to the observed data events. The distribution is fitted with a student's t function and a polynomial background function. The background is represented with the dashed line.

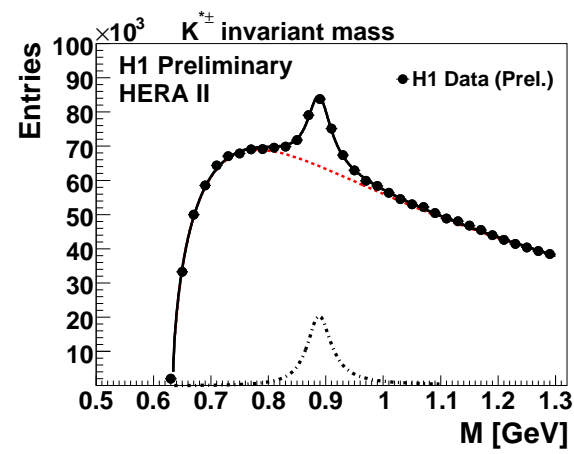

Figure 2: Invariant mass spectrum of $K_{S}^{0} \pi^{ \pm}$ combinations. The distribution is fitted with the sum of a relativistic Breit-Wigner function (solid line) and a background function (dashed line). The signal is shown with the dash-dotted line. 


\section{Results}

The total inclusive cross section for $K^{* \pm}$ in the visible range of $5<Q^{2}<100 \mathrm{GeV}^{2}$, $0.1<y<0.6, p_{T}\left(K^{* \pm}\right)>1 \mathrm{GeV},\left|\eta\left(K^{* \pm}\right)\right|<1.5$ is measured

$$
\sigma\left(e p \rightarrow e K^{* \pm} X\right)=7.36 \pm 0.087 \text { (stat.) } \pm 0.88 \text { (sys.) nb. }
$$

$K^{*+}$ and $K^{*-}$ cross sections which may indicate an asymmetry between $s$ and $\bar{s}$ distributions of the sea quarks in the proton are measured separately and give same result within the uncertainties. Differential cross sections for the $K^{* \pm}$ production are presented as a function of the event variables four momentum squared of the virtual photon $Q^{2}$ and the centre-of-mass energy of the hadronic final state $W_{\gamma p}$, and of transverse momentum $p_{T}$, pseudo-rapidity $\eta$, Feynman variable $x_{F}{ }^{\text {a }}$, the transverse momentum squared $P_{T}^{* 2}$ of the final state particles $K^{* \pm}$. The quantities $p_{T}, \eta$ and $Q^{2}$ were measured in the laboratory frame and $x_{F}, P_{T}^{* 2}$ and $W_{\gamma p}$ in the hadronic centre of mass system. The comparison between the measured inclusive differential cross sections to the leading order Monte Carlo (MC) predictions (DJANGOh1.4 [3] and RAPGAP3.1 [4]) is shown in Fig. 3. In general, both the colour dipole model (CDM, implemented in DJANGOh1.4) and matrix elements matched with parton showers (MEPS, implemented in RAPGAP3.1) provide a reasonable overall description of the data. However, differences are observed in some bins. In particular the $\eta\left(K^{* \pm}\right)$ distribution is not described by the models. A similar observation has been made in a recent measurement of the $\mathrm{H} 1$ experiment for other strange particles $\left(K_{S}^{0}\right.$ and $\Lambda$ ) [5]. It has also been observed that the CDM model describes the data slightly better than MEPS. The contributions of various quark flavors of the primarily incoming particles by the hard subprocess from the proton side are shown in Fig. 4 . In general $u$ and $d$ quarks give the largest contribution to the production of $K^{* \pm}$. The second largest contribution is from $c$ and $b$ quarks. Only about $20 \%$ of the contribution originates from $s$ quarks. The quark composition depends on $x_{F}$ : heavy quarks $(c$ and $b)$ are produced via boson gluon fusion (BGF) and contribute mainly at small $x_{F}$. The $s$ quarks are also produced via BGF, but their fraction rises with $x_{F}$.

\section{Conclusions}

$K^{*}(892)^{ \pm}$vector meson production observed through the decay chain $K^{*}(892)^{ \pm} \rightarrow K_{s}^{0} \pi^{ \pm}$, in neutral current deep-inelastic ep scattering has been measured for the first time at HERA. The inclusive production cross sections as a function of the event kinematics and final state variables are presented in the phase space of $5<Q^{2}<100 \mathrm{GeV}^{2}, 0.1<y<0.6$, $p_{T}\left(K^{* \pm}\right)>1 \mathrm{GeV}$, and $\left|\eta\left(K^{* \pm}\right)\right|<1.5$. The measurement of the $K^{* \pm}$ cross sections provide further insight in the fragmentation process and helps to disentangle uncertainties related to the strangeness suppression. The contributions of the different processes to the production of strange $K^{* \pm}$ mesons were investigated. For the separation of the different subprocesses, the flavour of the quark participating in the hard interaction was studied. Only $20 \%$ of the produced $K^{* \pm}$ mesons can be related to strangeness content of the proton.A rise of relative contribution of $s$ quark with increasing $x_{F}$ and $P_{T}^{* 2}$ was observed. In general, it is found that the overall features of the data are reproduced by both DJANGO and RAPGAP

\footnotetext{
aThe Feynman variable $x_{F}$ is defined as $x_{F}=2 \cdot p_{L} / W$, where $p_{L}$ is the momentum of $K^{* \pm}$ along the photon direction and $W$ is the centre of mass energy of the hadronic system.
} 
MC models. However, the predictions fail to describe the details of the distributions in various regions of the phase space, in particular in the low $p_{T}$ and $\eta$ regions. The strange to anti-strange quark production asymmetry has been studied by measuring $K^{*+}$ to $K^{*-}$ asymmetry. As a result, there has been no difference observed between $K^{*+}$ and $K^{*-}$ cross sections.
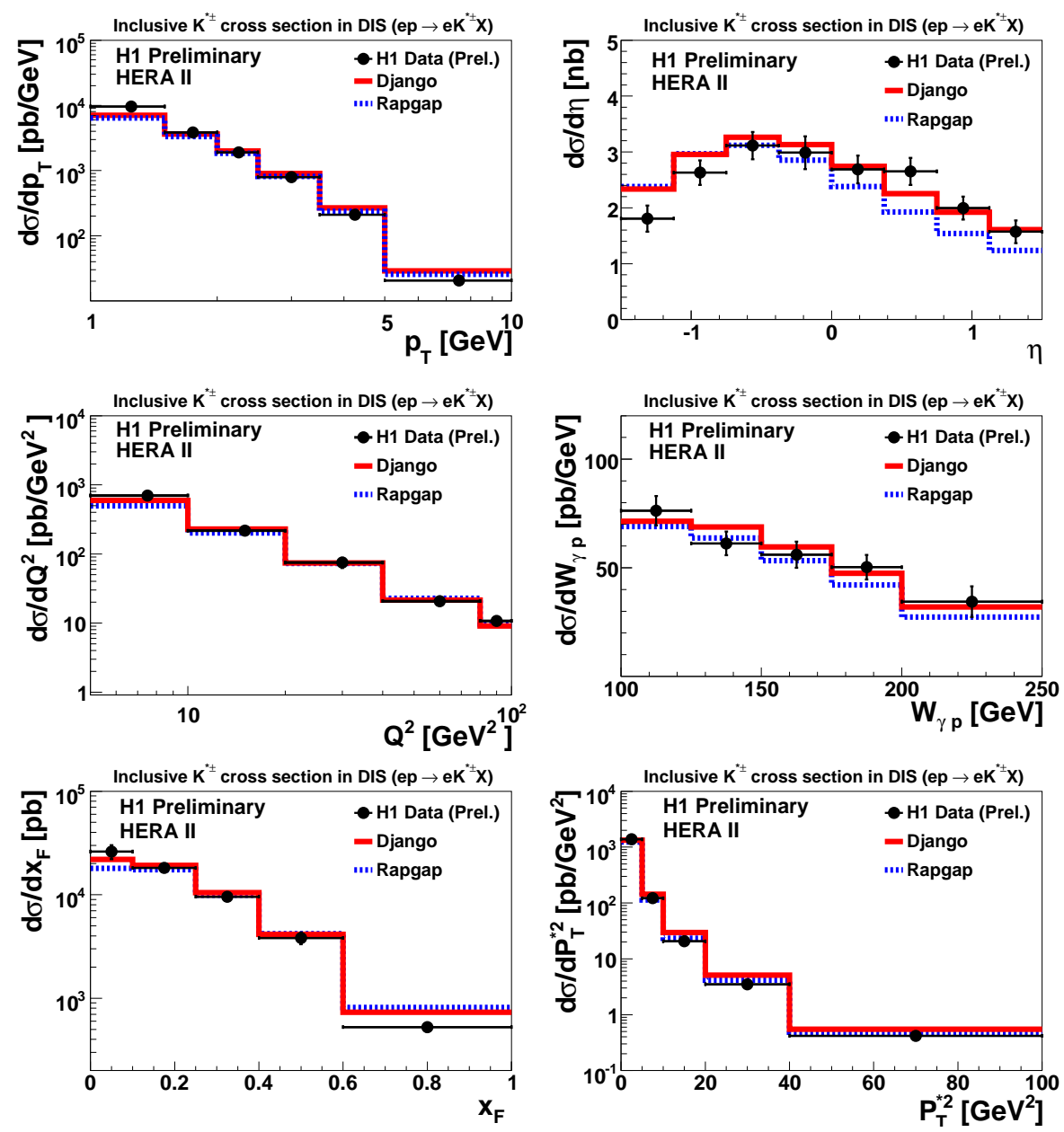

Figure 3: The measured inclusive differential cross $K^{* \pm}$ sections as a function of $p_{T}, \eta, Q^{2}$, $W_{\gamma p}, x_{F}$ and $P_{T}^{* 2}$. The inner errors in the figures represent the statistical error, while the outer is the statistical and the systematic errors added in quadrature. The measured cross section is compared to predictions from DJANGO (continuous line) and RAPGAP (dashed line). 

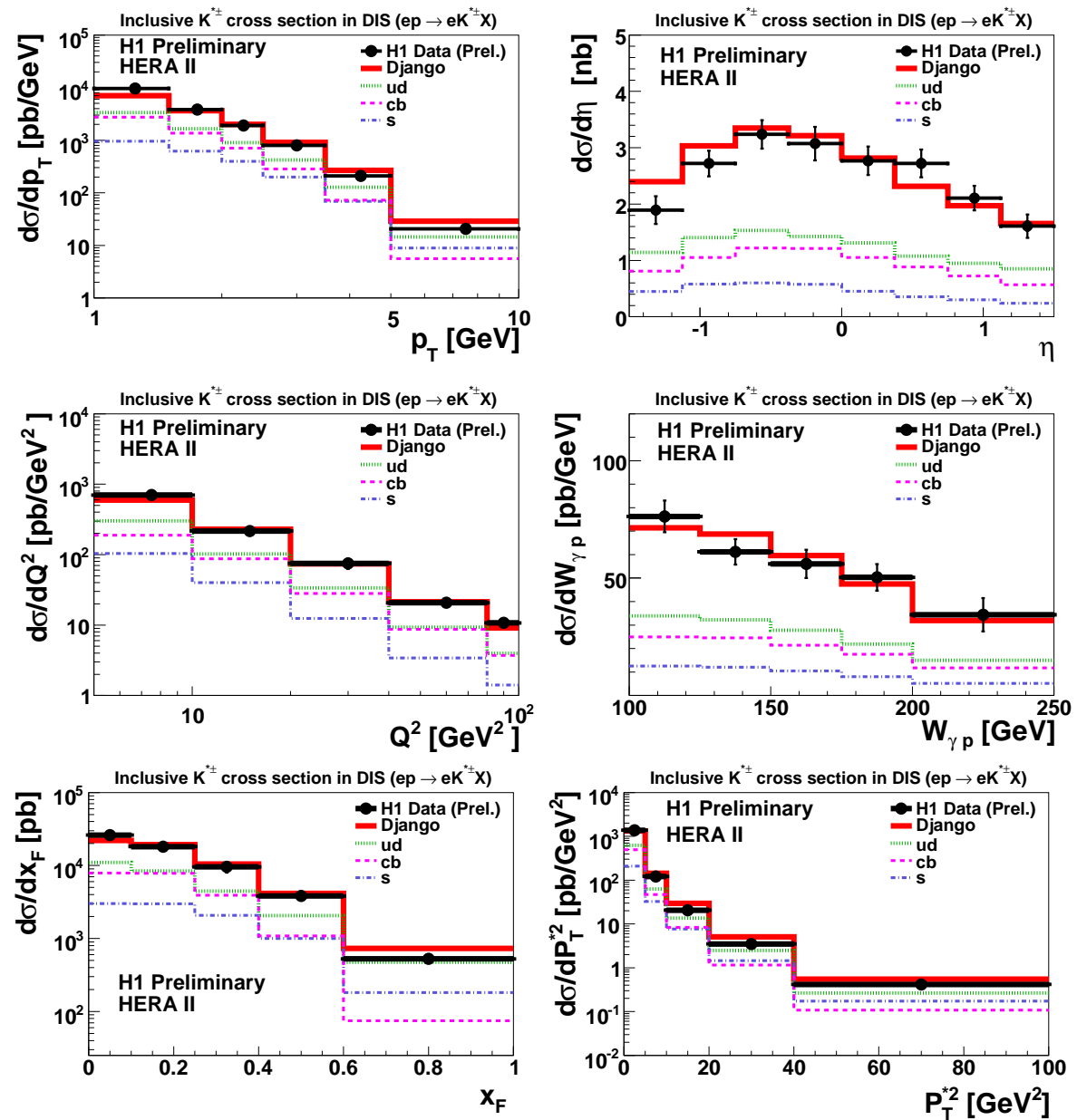

Figure 4: The measured inclusive differential $K^{* \pm}$ cross sections as a function of $p_{T}, \eta, Q^{2}$, $W_{\gamma p}, x_{F}$ and $P_{T}^{* 2}$. The inner errors in the figures represent the statistical error, while the outer errors are the statistical and the systematic errors added in quadrature. The quark contributions $u d$ (dotted lines), $c b$ (dashed lines), and $s$ (dash-dotted lines) are shown.

\section{References}

[1] Slides:

http: //indico. cern. ch/getFile.py/access? contribId=245\&sessionId=3\&resId=0\&materialId=slides\&conf Id=53294

[2] U. Bassler, G. Bernardi, Nucl. Instrum. Meth. A361, 197 (1995).

[3] G. A. Schuler and H. Spiesberger, Physics at HERA ed. by W. Buchmuller and G. Ingelman vol. 3 1419 (1992).

[4] H. Jung, Comp. Phys. Commun. 86147 (1995).

[5] F. D. Aaron et al., submitted to Eur. Phys. J. C, [arXiv:hep-ex/0810.4036v2] (2008). 